\title{
Pericardial Flap Interposition for the Definitive Management of Recurrent Tracheoesophageal Fistula
}

\author{
By Michael J. Wheatley and Arnold G. Coran \\ Ann Arbor, Michigan
}

\begin{abstract}
- From 1974 to 1988 , six children with fistula recurrence following primary tracheoesophageal fistula (TEF) repair have been managed at our hospital. Reclosure of the fistula with pleural flap interposition was used as the initial corrective procedure in five patients, with an $80 \%$ incidence of second TEF recurrences. Due to these discouraging results, we have abandoned this technique and instead favor fistula reclosure with interposition of a pedicle of vascularized pericardium between the esophageal and tracheal suture lines. The pericardial pedicle is easy to mobilize, effectively isolates the tracheal and esophageal suture lines, and eliminates the often difficult task of finding sufficient pleural tissue for fistula interposition in small infants. Furthermore, the vascularized pedicle serves as a template for the ingrowth of neomucosa from the existing esophageal mucosa should an anastomotic leak occur. Since adopting this approach, we have used this technique on four children, three of whom had second recurrences after pleural interposition, and have had no evidence of recurrent fistula formation at follow-up ranging from 2 to 6 years. With at least a $20 \%$ incidence of second recurrences reported following conventional management with fistula closure and pleural interposition, we believe use of a pericardial flap in the management of the recurrent TEF is the most appropriate surgical procedure.
\end{abstract}

Copyright $\odot 1992$ by W.B. Saunders Company

INDEX WORDS: Tracheoesophageal fistula, recurrent.

$\mathbf{P}$ ERHAPS the most vexing problem following primary repair of tracheoesophageal fistula (TEF) and esophageal atresia (EA) is recurrence of the TEF. Once thought to be a rare complication, TEF recurrence has a reported incidence between $5 \%$ and $11 \% .^{1-3}$ While the diagnosis of a recurrent fistula can be elusive, the surgical management is even more problematic with second recurrences not at all uncommon following fistula reclosure and pleural interposition. In 1986, we reported on the use of a pericardial flap to manage a child with a recurrent TEF. ${ }^{4}$ Over the past 3 years, this procedure has been used on four patients, three of whom had second recurrences after conventional surgical clo-

From the Section of Pediatric Surgery, C.S. Mott Children's Hospital and the University of Michigan Medical School, Ann Arbor, MI.

Presented at the 43rd Annual Meeting of the Surgical Section of the American Academy of Pediatrics, New Orlcans, Louisiana, October 26-27, 1991

Address reprint requests to Amold G. Coran, MD, C.S. Mott Children's Hospital, Room F7516, Box 0245, Ann Arbor, MI 48109.

Copyright $\odot 1992$ by W.B. Saunders Company

0022-3468/92/2708-0037\$03.00/0 sure of their recurrent fistula. It has proven to be an invaluable technique for managing this difficult complication.

\section{MATERIALS AND METHODS}

From January 1974 to December 1988, 80 neonates with EA or EA and TEF presented to the Mott Children's Hospital, and 67 were successfully managed with division of the TEF and a one-layer 5-0 silk end-to-end esophagoesophagostomy as a single or staged procedure. All repairs were done through a right retropleural thoracotomy. Placement of a Stamm feeding gastrostomy was performed in 59 of the 67 children either prior to or at the time of EA repair. During this interval, six patients developed a recurrent TEF. One child was asymptomatic and his fistula was noted on a routine postoperative barium study 1 week following the primary repair. The remaining five all had severe respiratory problems that mandated search for a recurrence. Diagnosis was made endoscopically in one child and with barium tube esophagograms in the remainder. Five of the six developed the recurrence within 3 months of their initial TEF repair and the sixth presented 23 months following her initial surgery with a respiratory arrest.

\section{RESULTS}

As the initial corrective procedure, five of the patients underwent reclosure of the fistula with interposition of a piece of pleural tissue to separate the esophageal and tracheal closures. Only one of these five children did well following surgery. This child had a pinpoint fistula that did not recur after the reclosure. The other four developed a second recurrence of their TEF, all within 5 months following initial reclosure (Fig 1). One of the four patients with a second recurrence died shortly after diagnosis from complications related to her multiple medical problems as well as to the TEF. The second recurrence in the remaining three children was managed with reexploration through a right transpleural thoracotomy, division of the TEF and mobilization of a vascularized pericardial flap with interposition between the esophageal and tracheal suture lines as described subsequently. All three have done well with no clinical or radiographic evidence of a recurrent fistula at follow-up ranging from 2 to 6 years (Fig 2). Based on our poor results with pleural interposition between the esophageal and tracheal suture lines, the sixth patient, the most recent infant with a first recurrence, was managed with fistula redivision and pericardial flap interposition as his initial corrective procedure and is doing well without any respiratory symptoms 2 years following this operation. 


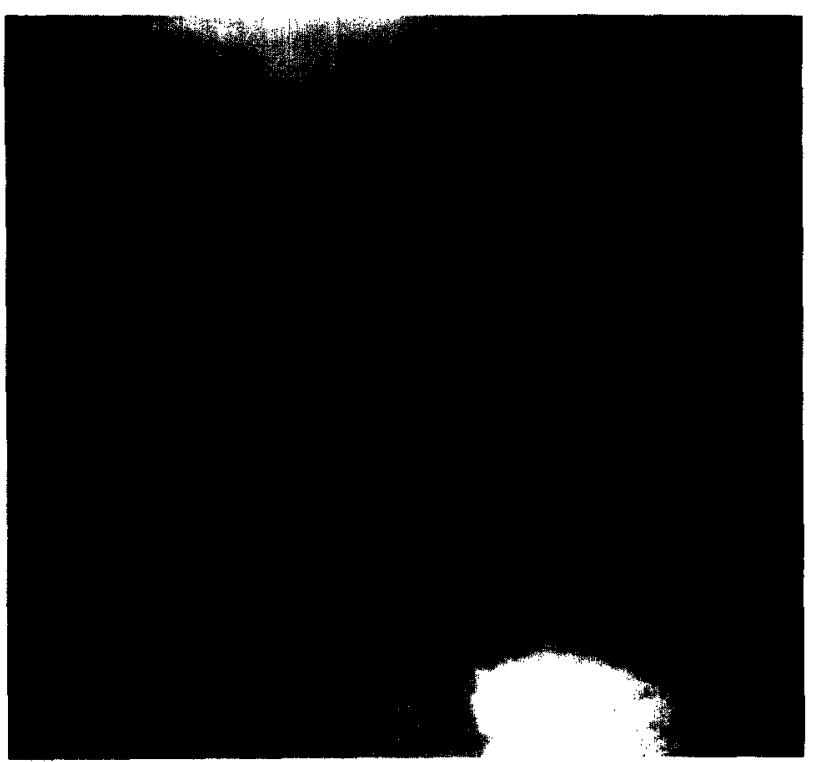

Fig 1. Barium esophagogram showing a second recurrence following fistula reclosure and pleural interposition for a TEF recurrence.

\section{Operative Technique}

Following diagnosis of a recurrent TEF, a nasojejunal tube is passed and the child is maintained on enteral nutrition for a period of 4 to 6 weeks both to improve nutritional status and to allow resolution of the inflammatory reaction in the area of the recurrent fistula. Following adequate weight gain, the patient is returned to the operating room and, if possible, the TEF is bronchoscopically visualized and stented with a no. 2 Fogarty catheter to aid in intraoperative

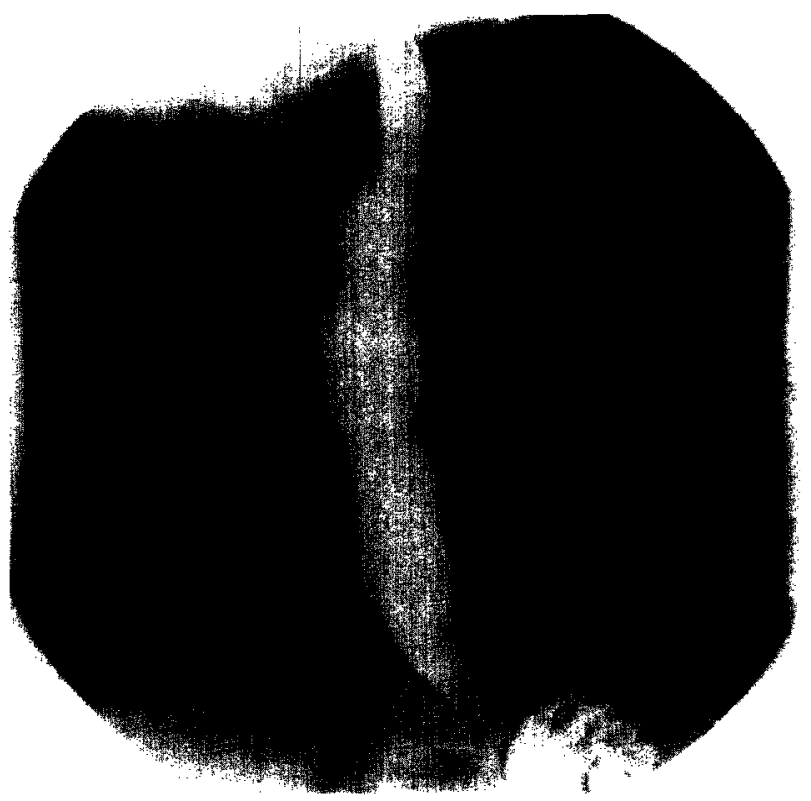

Fig 2. Barium esophagogram in the same patient 2 years following fistula reclosure with interposition of a pericardial flap showing no recurrence of the fistula. localization (Fig 3). A right posterolateral transpleural thoracotomy is performed and the proximal and distal esophagus isolated. With the aid of the stent, the fistula is resected lcaving a cuff of esophagcal tissue on the membranous trachea for closure (Fig 4). Both the esophagus and trachea are closed with single-layer of interrupted 5-0 cardiovascular silk stitches. Following closure of the fistula, the right phrenic nerve is mobilized off the pericardium from the midpoint of the superior vena cava (SVC) to a level just below the inferior margin of the right atrium. Transverse incisions are then made in the pericardium $1 \mathrm{~cm}$ above and below the mobilized margins of the phrenic nerve and carried anteriorly across the right atrium. The transverse incisions are then joined by a vertical incision, creating a vascularized pedicle of pericardium with its base attached to the SVC and the right atrium (Fig 5). The pedicle of pericardium is then brought under the right phrenic nerve and interposed between the tracheal and esophageal suture lines and secured in place with interrupted 4-0 silk sutures (Fig 6). The pericardial defect overlying the right atrium is closed looscly with interrupted 4-0 silk sutures and a chest tube is placed in the right pleural space. The chest is then closed in layers. A routine barium esophagogram is obtained on the 7 th postoperative day.

\section{DISCUSSION}

TEF recurrence following primary $\mathrm{EA}$ and $\mathrm{TEF}$ repair in infancy is not a rare complication, with an incidence of $9 \%$ in our series of 67 patients. Documentation of a recurrence can be challenging, and, although barium esophagogram and endoscopy were diagnostic in our group, these studies are not particularly sensitive and can be misleading in failing to identify a tracheoesophageal communication. ${ }^{3}$ This diagnosis must be considered and reconsidered in the evaluation of any postoperative EA child with persis-

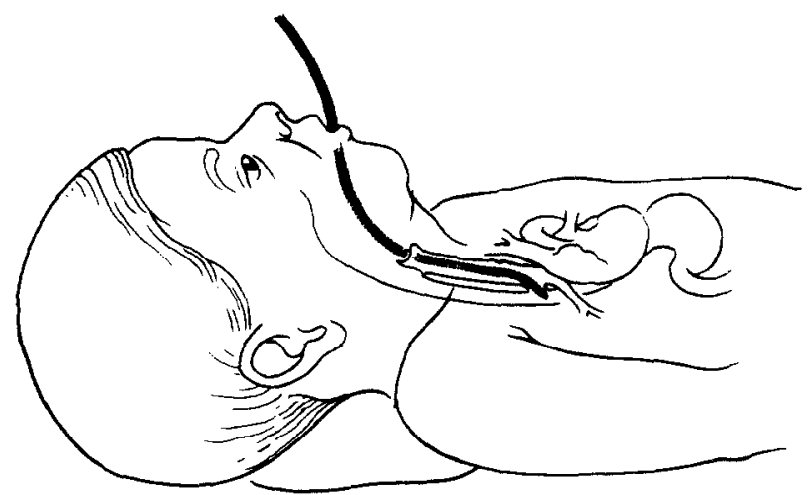

Fig 3. Preoperative identification of the fistula bronchoscopically allows placement of a Fogarty catheter through the fistula to act as a stent to aid in operative identification of the fistula. 


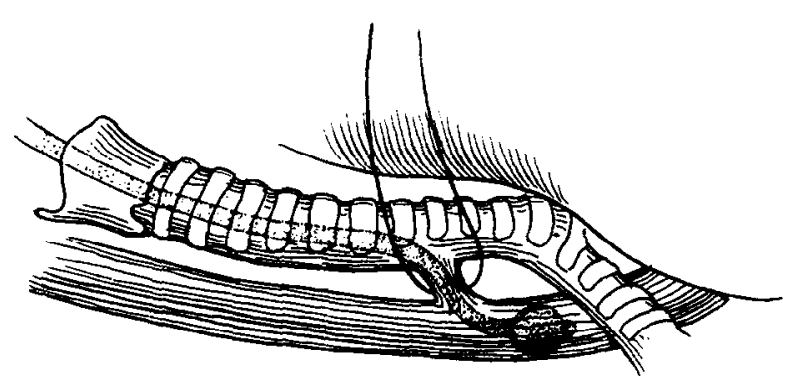

Fig 4. With the aid of the Fogarty stent, the fistula is isolated and surrounded with an 0 -silk tie.

tent respiratory symptoms because not all postoperative pulmonary problems in this setting are due to gastroesophageal reflux or aspiration. ${ }^{5} \mathrm{~A}$ variety of radiologic techniques have been described to assist in diagnosing the recurrent TEF, including cine barium swallow, right lateral decubitus esophagograms, and prone esophagrams. ${ }^{6,7}$ Like others, we have found the tube esophagogram, in which barium is injected under some pressure into a nasogastric tube as the tube is slowly pulled from the stomach to the esophagus, to be an essential part of the examination for a recurrent fistula. ${ }^{3}$ Repeated barium studies should be performed as long as recurrent TEF is suspected and until the diagnosis is made. This is particularly true in children with persistent or recurrent pulmonary symptoms following closure of a recurrent TEF as second recurrences can occur. In our series, pulmonary symptoms resolved in all patients following definitive repair, suggesting that persistent symptoms indicate an ongoing anatomic abnormality and are not simply the inevitable pulmonary sequela of this disorder.

Because the etiology of TEF recurrence is not well understood, prevention is a difficult task. Although the end-to-side technique appears to lead to a higher incidence of recurrent fistulization, ${ }^{8,9}$ the current series as well as others in which the end-to-end technique was primarily or exclusively used have a relatively constant recurrence rate near $10 \% .^{1-3,8,10,11}$

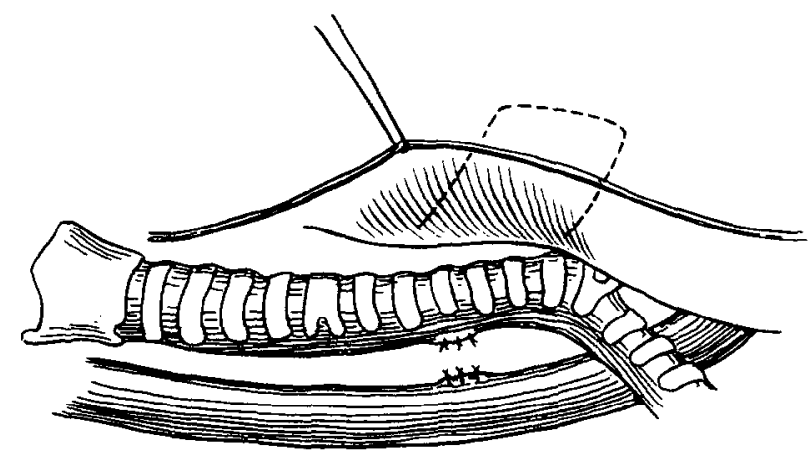

Fig 5. A vascularized pedicle of pericardium with its base attached to the SVC and right atrium is created.

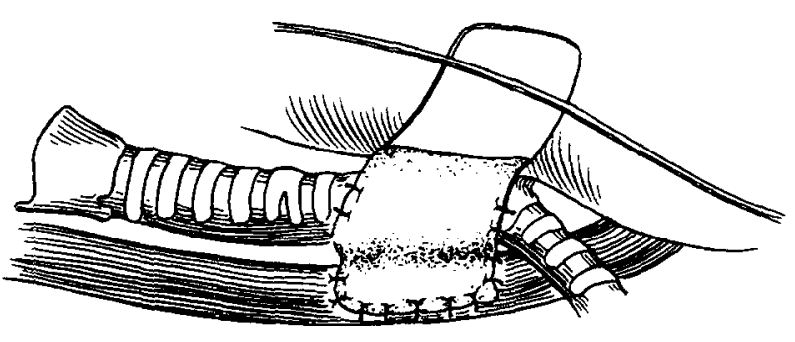

Fig 6. Pericardial pedicle is brought under the phrenic nerve then interposed between the tracheal and esophageal suture lines, where it is sewn in place.

Anastomotic tension during the primary repair and postoperative anastomotic leakage are thought to predispose to fistula formation, ${ }^{3}$ although only $50 \%$ of the patients with recurrences had anastomotic leakage and only one anastomosis was performed under tension. In our experience, careful fistula closure with suturing of the membranous trachea and use of pleural or intercostal muscle pedicle grafts have done little to impact on the incidence of recurrence.

Once diagnosed, surgical closure of a recurrent fistula is mandatory as spontaneous closure is a very rare occurrence, $2,3,11,12$ and delays in surgical closure place the child at risk for ongoing respiratory problems. As initially suggested by Haight, ${ }^{13}$ a 4- to 6-week waiting period prior to repair is beneficial because early operative correction may be technically difficult due to the marked inflammatory response often initially found in the area of the fistula. Nasojejunal tube feeding facilitates this temporizing approach and allows the maintenance of adequate nutrition without esophageal bypass and gastrostomy placement.

The surgical management of this complication has evolved over the past 7 years. Our first five patients initially underwent division and reclosure of the fistula with interposition of a pleural flap. One child with a pinpoint fistula did well with this approach, but the remaining four developed a second recurrence. Our experience is paralleled by Ein et al, who noted a $22 \%$ incidence of second recurrences following pleural flap interposition. ${ }^{3}$ One child with a second recurrence died prior to corrective surgery but the remaining three and one additional child with a primary recurrence have all done well following takedown of their recurrent fistula and interposition of a vascularized pericardial flap. Postoperative recovery was unremarkable in each instance, and, at follow-up ranging from 2 to 6 years, no recurrent fistulas have occurred.

We consider pericardial flap interposition with fistula division to be the procedure of choice for 
recurrent TEF. The vascularized pedicle of pericardium effectively isolates the tracheal and esophageal suture lines and eliminates the often difficult task of finding sufficient pleural tissue for fistula interposition in small infants. Furthermore, the vascularized pedicle serves as a template for the ingrowth of neomucosa from the existing esophageal mucosa should an anastomotic leak occur. ${ }^{14}$ Since the inci- dence of an initial fistula recurrence is nearly $10 \%$, the routine use of a pericardial interposition flap during primary EA repair is not unreasonable. However, with at least a $20 \%$ incidence of second recurrences reported following conventional management with fistula closure and pleural interposition, we believe use of a pericardial flap in the management of the recurrent TEF is appropriate.

\section{REFERENCES}

1. Manning PB, Morgan RA, Coran AG, et al: Fifty years experience with esophageal atresia and tracheoesophageal fistula. Ann Surg 204:446-453, 1986

2. Kafrouni G, Baick CH, Wooley MM: Recurrent tracheoesophagcal fistula; a diagnostic problem. Surgery 68:889-894, 1970

3. Ein SH, Stringer DA, Stephens CA, et al: Recurrent tracheoesophageal fistulas seventeen-year review. J Pediatr Surg 18:436441,1983

4. Botham MJ, Coran AG: The use of pericardium for the management of recurrent tracheoesophageal fistula. J Pediatr Surg 21:164-166, 1986

5. Delius R, Wheatley MJ, Coran AG, et al: Respiratory complications following tracheoesophageal fistula repair. Surgery (in press)

6. Cumming WA: Esophageal atresia and tracheoesophageal fistula. Radiol Clin North Am 13:277-295, 1975

7. Thomas PS, Chrispin AR: Congenital tracheo-oesophageal fistula without oesophageal atresia. Clin Radiol 20:371-374, 1969

8. Daum R: Postoperative complications following operation for oesophageal atresia and tracheo-oesophageal fistula, in Rickham PP, Hecker WC, Prevot J (eds): Progress in Pediatric Surgery. Baltimore, MD, University Park Press, 1971, pp 209-237

9. Ein SH, Theman TE: A comparison of the results of primary repair of esophageal atresia with tracheoesophageal fistulas using end-to-side and end-to-end anastomoses. J Pediatr Surg 8:641-645. 1973

10. Filston HC, Rankin JC, Kirks DR: The diagnosis of primary and recurrent tracheoesophageal fistulas: Value of selective catheterization. I Pediatr Surg 17:144-148, 1982

11. Slim MS, Tabry IF: Left extrapleural approach for the repair of recurrent tracheo-esophageal fistula. J Thorac Cardiovasc Surg 68:654-657, 1974

12. Falletia GP: Recommunication on repair of congenital tracheoesophageal fistula. Arch Surg 88:779-786, 1964

13. Haight C: The esophagus, in Mustard WT, Ravitch MM, Snyder WH, et al (eds): Pediatric Surgery (ed 2). Chicago, IL, Year Book, 1979

14. Coran AG: Pericardioesophagoplasty: A new operation for partial esophageal replacement. Am J Surg 125:294-298, 1973

\section{Discussion}

Howard Filston (Knoxville, TN): Dr Coran and his associates have correctly, I believe, pointed out the $10 \%$ recurrence rate after TEF repair, which has been a rather universal experience. However, the high incidence of second recurrences is somewhat startling. They have offered a nice technique for solving the problem of finding something to interpose between the ligated ends of the TEF.

I would question whether their high rate of second recurrence is due to missing the fistula at operation for the first recurrence. They mention in the manuscript trying to stent the fistula preoperatively through the bronchoscope at the second recurrence. Did they stent the original recurrence for definitive identification of the fistula at that operation?

I find it very difficult to be certain that I have found these often tiny recurrent fistulas, and sometimes they are not where you expect them to be. Without such a catheter spanning the fistula, I find it very difficult to be certain that I have really found it and divided it.

Even identifying and confirming the presence of the recurrent fistula by bronchoscopy can be very difficult, let alone successfully passing a catheter through it. A technique of directly probing and cannulating recurrent fistulas during a pullout esophagography was developed by Dr Donald Kirks when he was at Duke. He used catheters made especially for the purpose of cannulating these fistulas.

I would ask three questions. How often did you cannulate the fistula at the first recurrence, and how often at the second recurrence?

You advise 4 to 6 weeks of nasojejunal feedings between discovery of the recurrence and operation. Do you keep the patients hospitalized the whole time? If so, this may not be truly cost-effective.

Finally, why don't you fold the flap all the way underneath the trachea? It looks to me from the drawing as though it only partially separates the trachea from the esophagus, and I couldn't really tell how it fits between the two closed fistula sites.

I like the pericardial flap idea, but would recommend that it is well worth the effort to have the recurrent fistula cannulated at the time of reoperation.

Jerrold Becker (New York, NY): In 1982, Dr Ein presented the experience at Toronto with recurrent 
TEF, and in discussing that paper I mentioned that I felt that a recurrent TEF is a preventable disease.

If one ligates and divides the azygous vein laterally, and then at the end of the anastomosis interposes it by covering the tracheal closure with it and just suturing the vein to the endothoracic fascia, you can prevent recurrent TEF.

Arnold G. Coran (response): First of all, Howard, we always stent them when possible. On the first go-around, about $90 \%$ of them were stented. On the second go-around, it was more difficult because of the circuitous nature of the fistula, and we couldn't stent them all. We were only stenting about $50 \%$ of the cases.
As far as the question of hospitalization is concerned, the last one of these was done 5 years ago, and at that time we weren't able to have the kind of home nutritional support that we carry out today. I think today, if we had a child on nasojejunal feeding, we would send the child home and have them managed by a visiting nurse.

As far as the diagram on coverage is concerned, the pericardial flap comes down, and it's hard to show in the diagram, but it goes inside that groove between the trachea and the esophagus. It's sitting way around the corner. It doesn't look like that as well in the diagram, but it's brought all the way inside there to cover it. 